\title{
Understanding Donor-Acceptor Interfaces In Organic Photovoltaics Using Analytical Transmission Electron Microscopy.
}

\author{
James Gilchrist $^{1}$, Toby Basey-Fisher ${ }^{1}$, Sandrine Heutz ${ }^{1}$ and David W. McComb ${ }^{2}$ \\ 1. Imperial College London, Department of Materials, Exhibition Road, London, SW7 2AZ, UK \\ 2. The Ohio State University, Department of Materials Science and Engineering, Columbus, Ohio 43210, \\ USA
}

Organic photovoltaic devices could potentially provide a cheap and lightweight source of energy [1]. However, processes taking place that are important for the efficient generation of electric power, are dramatically affected by the morphology, microstructure and the electronic energy levels of the films and interfaces that comprise these devices. This complexity provides a fascinating research challenge, one which the transmission electron microscope (TEM), when combined with the variety of techniques available, can tackle. However, problems arising from damage due to sample preparation and electron beam exposure have previously made these materials problematic to work with.

A low damage method for preparing cross sections of device structures, using a focused ion beam (FIB) to remove and thin a sample to electron transparency, has been developed which allows us to observe the crystalline nature of copper phthalocyanine $(\mathrm{CuPc})$ and buckminsterfullerene $\left(\mathrm{C}_{60}\right)$ thin films and heterostructures, a common donor-acceptor system [2]. This has allowed detailed information about molecular orientation to be to be obtained from films, as well as the important donor-acceptor interface, to be collected. The lattice fringes of the $\mathrm{CuPc}$ (100) plane are visible in the three types of device structure analysed. Using this fringe the molecular orientation of the CuPc molecules can be identified [3]. Since charge transport in $\mathrm{CuPc}$ is dependent on molecular orientation, this information is highly relevant to device efficiency. Similarly, lattice fringes are observed within the $\mathrm{C}_{60}$ film, which result from diffraction from the (111), (220) and (311) planes. A preferential orientation of the $\mathrm{C}_{60}$ crystals is discovered within the bilayer device structure, where the $\mathrm{C}_{60}(111)$ plane lies parallel to the $\mathrm{CuPc}(100)$ fringes (figure 1).

It is however with the advent of the new high-efficiency collection and detection energy dispersive $\mathrm{x}$-ray (EDX) analysis that these systems can be studied with unprecedented detail. By using scanning transmission electron microscopy (STEM), EDX spectra are recorded as a function of cross section area and parameters such as interface roughness, molecular and elemental diffusion can be extracted from bilayer device structures (figure 2). Also, when analyzing the co-deposited layer in the bulk heterojunction device structure, no segregation can be observed, suggesting that there is no large scale separation of the donor or acceptor materials. These parameters are vital for the efficient generation of current, and the characterisation techniques described could potentially allow rapid advances in the understanding and optimisation of organic electronic devices. 
[1] Forrest, S. R. The path to ubiquitous and low-cost organic electronic appliances on plastic. Nature 428, 911-8 (2004).

[2] Sullivan, P., Jones, T. S., Ferguson, a. J. \& Heutz, S. Structural templating as a route to improved photovoltaic performance in copper phthalocyanine/fullerene $\left(\mathrm{C}_{60}\right)$ heterojunctions. Applied Physics Letters 91, 233114 (2007).

[3] Gilchrist, J. B., Heutz, S. \& McComb, D. W. Using TEM and XRD to probe crystal orientation in organic thin films grown with OMBD. Journal of Physics: Conference Series 371, 012042 (2012).

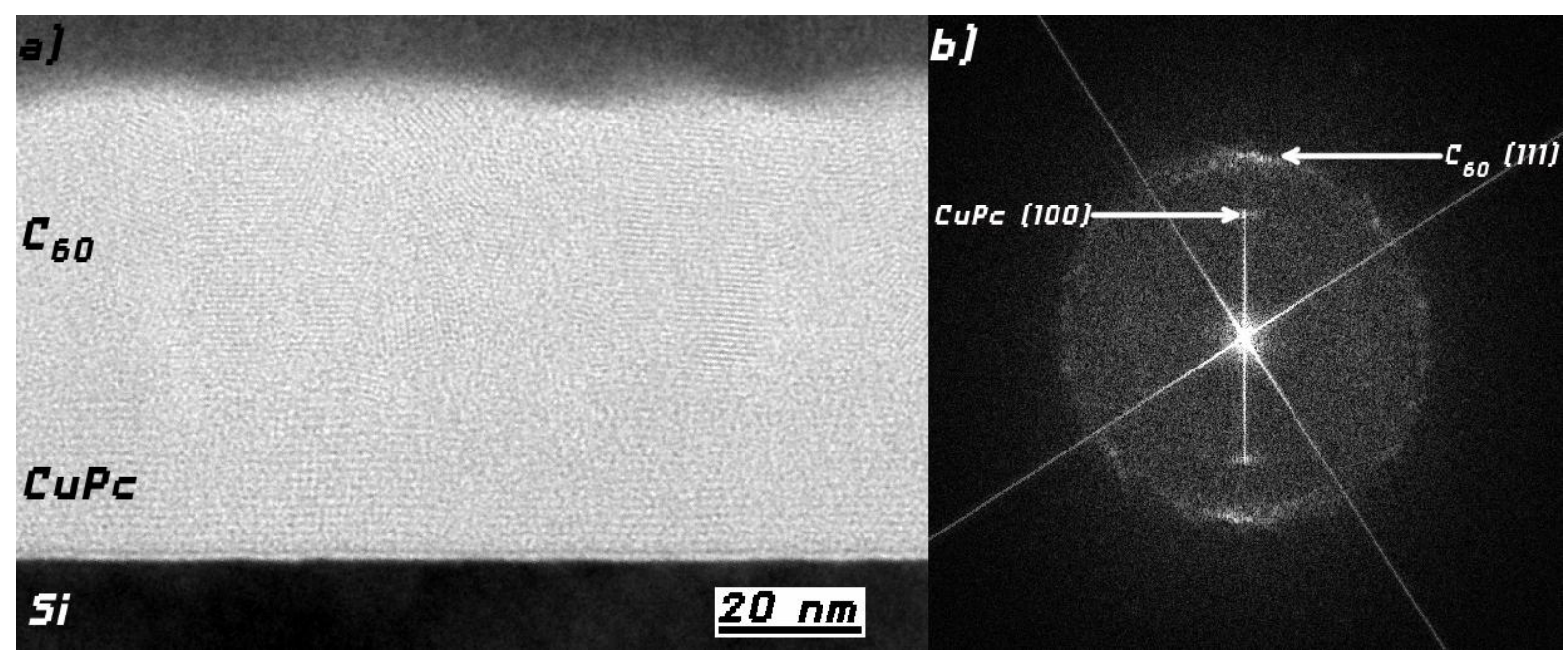

Figure 1. a) $\mathrm{BF}$ TEM image of a $\mathrm{CuPc} / \mathrm{C}_{60}$ bilayer. b) $\mathrm{FFT}$ of the image in a) with the spatial frequencies labeled, note the increased intensity of the $\mathrm{C}_{60}$ (111) frequency when inline with the CuPc (100) frequency

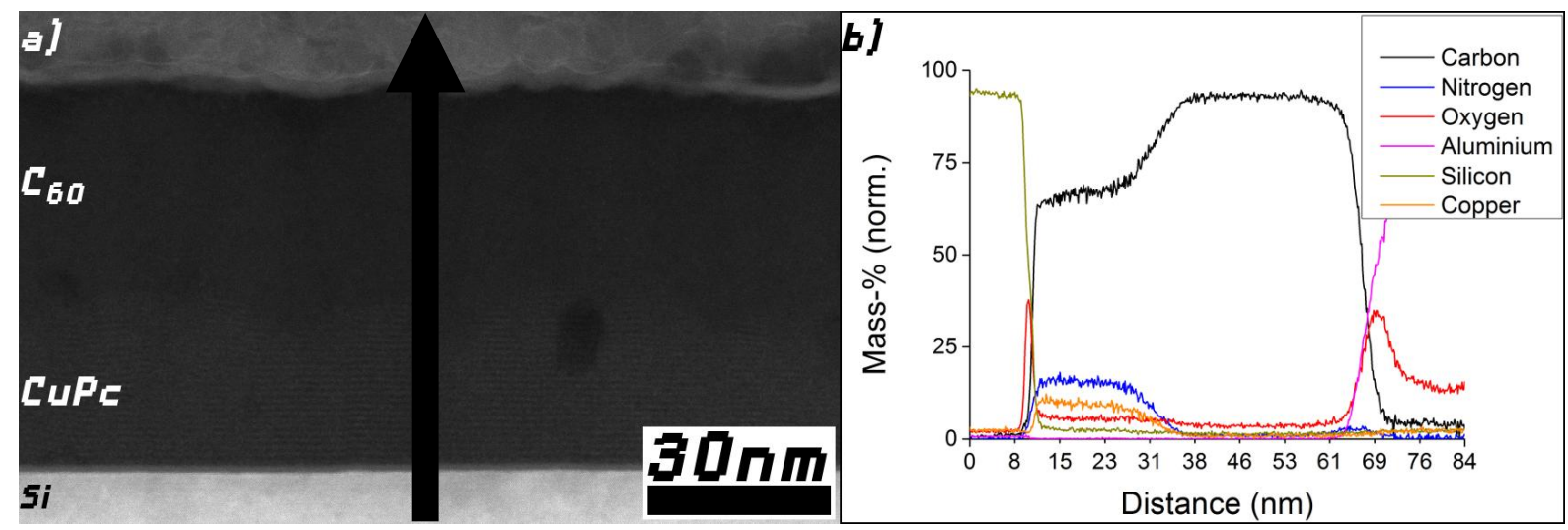

Figure 2. a) HAADF STEM image of $\mathrm{CuPc} / \mathrm{C}_{60}$ bilayer showing the direction that linescan $\mathrm{b}$ ) has been taken 